\title{
Glutamine supplementation in bone marrow transplantation
}

\author{
Thomas R. Ziegler* \\ Department of Medicine, Division of Endocrinology and Metabolism, Emory University School of Medicine, \\ Atlanta, GA 30322, USA
}

\begin{abstract}
An increasing number of clinical investigations have focused on supplementation of specialized enteral and parenteral nutrition with the amino acid glutamine. This interest derives from strong evidence in animal models and emerging clinical data on the efficacy of glutamine administration following chemotherapy, trauma, sepsis and other catabolic conditions. Glutamine has proteinanabolic effects in stressed patients and, among many key metabolic functions, is used as a major fuel/substrate by cells of the gastrointestinal epithelium and the immune system. These effects may be particularly advantageous in patients undergoing bone marrow transplantation (BMT), who exhibit post-transplant body protein wasting, gut mucosal injury and immunodeficiency. Studies to date indicate that enteral and parenteral glutamine supplementation is well tolerated and potentially efficacious after high-dose chemotherapy or BMT for cancer treatment. Although not all studies demonstrate benefits, sufficient positive data have been published to suggest that this nutrient should be considered as adjunctive metabolic support of some individuals undergoing marrow transplant. However, BMT is a rapidly evolving clinical procedure with regard to the conditioning and supportive protocols utilized. Thus, additional randomized, double-blind, controlled clinical trials are indicated to define the efficacy of glutamine with current BMT regimens.
\end{abstract}

Glutamine: Bone marrow transplantation: Mucositis

\section{Introduction}

Use of bone marrow transplantation (BMT) to treat malignant and non-malignant disease has increased dramatically during the past decade (Mogul, 2000; Slavin, 2000). Patients may undergo allogeneic BMT, in which cells for transplant are derived from HLA-matched donors, or autologous BMT, in which cells for transplant are derived from the patient. The most commonly used method to obtain cells for BMT is ex vivo mobilization of stem cells from peripheral blood using colony-stimulating growth factors (Mogul, 2000; Slavin, 2000). Administration of high doses of chemotherapy (often including bulsulfan, cyclophosphamide, and/or epoposide), with or without total body irradiation (TBI), is a feature of most BMT protocols for malignancy. These regimens are designed to ablate the underlying malignant cells, but may cause adverse gastrointestinal complications including nausea, vomiting, inflammation of the oral and esophageal mucosa (mucositis), abdominal pain and diarrhea (Blijlevens et al. 2000; Jonas et al. 2000). Nonmyeloablative stem cell allogeneic transplantation (NST) is also undergoing clinical investigation for malignant and non-malignant disease (e.g. induction of organ allograft tolerance or genetic disorders) and is associated with less gastrointestinal toxicity (Slavin, 2000). However, for malignant diseases, the efficacy of NST remains to be defined and high-dose cytotoxic therapy remains the standard of care in BMT protocols (Mogul, 2000; Slavin, 2000).

Adverse gastrointestinal effects associated with highdose chemotherapy given prior to BMT may decrease spontaneous food intake, disrupt the gastrointestinal mucosal barrier and interfere with nutrient transport and absorption (Blijlevens et al. 2000). In addition, marked body protein wasting, immune system suppression and oxidative stress occur after BMT, despite administration of conventional nutrition support (Mogul, 2000; Slavin, 2000; Jonas et al. 2000; Durken et al. 1995). Although parenteral nutrition (PN) and oral nutrient supplements are commonly prescribed, the efficacy of nutrition support in BMT patients still remains unclear (Weisdorf et al. 1987; Ziegler et al. 1990; Klein \& Koretz, 1994). This is due to the small number of well-designed randomized clinical trials and rapid changing conditioning and growth factor regimens, which makes controlled studies of nutrition support

\footnotetext{
Abbreviations: BMT, bone marrow transplantation; TBI, total body irradiation; NST, non-myeloablative stem cell allogeneic transplantation; PN, parenteral nutrition.
}

* Corresponding author: Dr Thomas R. Ziegler, M.D., fax +1 404727 5563, email tzieg01@emory.edu 
difficult. Thus, administration of specialized nutrition varies widely among BMT centers worldwide.

An increasing number of clinical investigations have focused on supplementation of specialized enteral and parenteral nutrition with the amino acid glutamine to improve the efficacy of nutritional support (reviewed in Darmaun, 2000; Griffiths, 1999; Ziegler et al. 2000a; Wilmore \& Shabert, 1998; Ziegler et al. 2000b). This interest derives from strong evidence in animal models and an increasing number of clinical trials in cancer and noncancer patients demonstrating benefits of glutamine supplementation in certain catabolic states, including patients following chemotherapy, trauma and sepsis. Glutamine has protein-anabolic effects and is used as a major fuel or substrate by cells of both the gut epithelium and the immune system (Darmaun, 2000; Griffiths, 1999; Ziegler et al. 2000a; Wilmore \& Shabert, 1998; Ziegler et al. 2000b). These effects may be particularly advantageous in patients undergoing BMT, who exhibit body protein wasting, immunodeficiency, mucositis and gut mucosal injury. In critically ill and post-operative patients, nutritional formulations supplemented with either free L-glutamine or glutamine dipeptides (glycyl-glutamine or alanyl-glutamine) have been shown to improve nitrogen balance, decrease the incidence of infection, diminish length of hospital stay, improve immune functions and reduce hospital costs (Table 1). This review will summarize available clinical literature on adjunctive use of glutamine supplementation in patients undergoing BMT. Additional published reports on glutamine use in non-BMT patients receiving high-dose chemotherapy will also be reviewed.

\section{Studies on intravenous administration of glutamine in BMT}

The initial clinical safety and efficacy of glutamine administration in BMT patients were reported in 1990 and 1992, respectively (Ziegler et al. 1990; Ziegler et al. 1992). Following studies in healthy adult subjects, tolerance of L-glutamine-containing PN at doses of 0.285 and $0.570 \mathrm{~g} / \mathrm{kg}$ per day was demonstrated when given for several weeks in adults undergoing allogenic BMT (Ziegler et al. 1990). After clinical safety and dose-related protein-anabolic

Table 1. Beneficial effects of enteral or parenteral administration of L-glutamine or glutamine dipeptides reported in double-blind, prospective randomized trials in non-cancer patients

- Maintained plasma glutamine levels, improved nitrogen balance and enhanced skeletal muscle protein synthetic rates (postoperative patients)

- Increased D-xylose absorption (critically ill patients)

- Improved circulating immune cell number and function (postoperative and critically ill patients)

- Decreased length of hospital stay (postoperative patients)

- Decreased requirements for mechanical ventilation (very low birthweight infants)

- Decreased incidence of hospital infection (trauma patients, very low birthweight infants)

- Decreased cost of hospital care (postoperative and critically ill patients) effects were demonstrated, a double-blind, randomized controlled trial was performed in forty-five clinical patients undergoing allogeneic BMT for hematological malignancies (Ziegler et al. 1992). Patients received either glutamine-free PN per standard protocols $(n=21)$ or isocaloric, isonitrogenous, L-glutamine-supplemented PN $(n=24)$. The PN formulas were designed to providing estimated requirements for energy (basal energy expenditure $+50 \%)$ and protein $(1.5 \mathrm{~g} / \mathrm{kg}$ per day) and were supplemented with L-glutamine at $0.57 \mathrm{~g} / \mathrm{kg}$ per day. $\mathrm{PN}$ in each group provided adequate amounts of essential amino acids for stressed patients. The amount of essential amino acids in the glutamine-containing solution was $\approx 30 \%$ less than in the control solution (33\% of total amino acids versus $45 \%$ ). Thus, the glutamine-enriched PN solution contained proportionally more non-essential amino acids, including L-glutamine, than the control PN. The two PN formulas contained equivalent amounts of branched-chain amino acids, but the control PN solution contained twice the amount of arginine as the experimental PN solution (Ziegler et al. 1992).

Following conditioning with high-dose chemotherapy and, in most cases TBI, BMT was performed and PN started the following day. Plasma glutamine concentrations rose $\approx 40 \%$ with glutamine-enriched PN, plasma glutamate, ammonia and pyroglutamic acid levels were unaltered and no adverse clinical or significant biochemical effects were noted during the 26-28 days of PN therapy (Ziegler et al. 1992). Daily nitrogen balance performed in the initial twenty-three patients between days 4 and 11 post-BMT was significantly improved with glutamine supplementation (glutamine $-1.4 \pm 0.5 \mathrm{~g}$ /day versus control $-4.2 \pm 1.2 \mathrm{~g} /$ day; $P=0.002)$ Although both groups demonstrated net body nitrogen loss, a significant

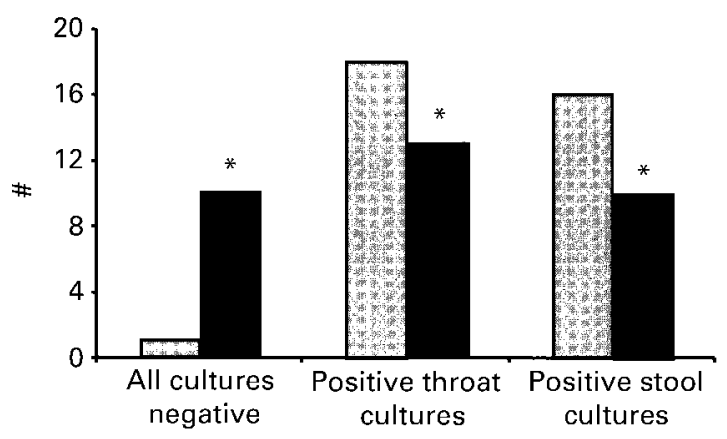

Fig. 1. L-Glutamine-supplemented parenteral nutrition decreased the incidence of positive microbial cultures after allogeneic bone marrow transplantation. Adult patients undergoing allogeneic bone marrow transplantation (BMT) for hematological malignancies received standard glutamine-free parenteral nutrition $(\square)$ or isocaloric, isonitrogenous, parenteral nutrition providing L-glutamine (ם) $(0.57 \mathrm{~g} / \mathrm{kg}$ per day). All patients underwent microbial decontamination with oral antibiotics during conditioning prior to BMT. Microbial cultures of blood, throat and stool were then obtained twice weekly and as clinically indicated until recontamination (discontinuation of oral antibiotics and administration of oral lactobacilli). In glutaminetreated patients, $42 \%$ remained culture negative, compared to only $5 \%$ in the control group. Significantly more standard PN than glutamine-supplemented patients developed one or more positive throat cultures (86\% v. $54 \%, P<0.05)$ and stool cultures $(75 \%$ v. $42 \%$, $P<0.05)$. ${ }^{*} P<0.04$ control $v$. glutamine-supplemented. 
improvement in 7-day cumulative nitrogen balance occurred with glutamine administration (glutamine $-9.7 \pm 3.4 \mathrm{~g}$ versus control $-29.6 \pm 8.6 \mathrm{~g} ; \quad P=0.002)$. The urinary excretion ratio of 3-methylhistidine/creatinine was significantly decreased with glutamine $(10.9 \pm 0.4$ versus $13.3 \pm 0 \cdot 9 ; P=0.03)$, suggesting that diminished myofibrillar protein breakdown was a potential mechanism for the protein-anabolic effect (Ziegler et al. 1992).

All patients underwent decontamination with oral antibiotics during conditioning; microbial cultures of blood, throat and stool were then obtained twice weekly and as clinically indicated until the time of microbial recontamination (discontinuation of oral antibiotics and administration of oral lactobacilli). Tabulation of culture results revealed that the incidence of total positive microbial cultures were significantly decreased in the glutamine group (Fig. 1). There was no difference between groups for neutrophil engraftment, incidence or severity of fever, oral mucositis or acute graft-versus-host disease, use of antibiotics or 100-day survival. However, as can be seen from Fig. 2 significantly fewer clinical infections developed in glutamine-treated patients and their length of hospital stay was significantly decreased, by 7 days versus controls (Ziegler et al. 1992).

Additional benefits from the use of glutamine supplementation demonstrated in this study, included attenuation of stress-induced extracellular fluid expansion (Scheltinga et al. 1991), enhanced recovery of circulating T-lymphocyte subsets within the first few weeks after hospital discharge (Ziegler et al. 1998) and improvement in mood scores (Young et al. 1993). The cost of hospital care was significantly decreased with glutamine supplementation in this group of BMT patients, primarily due to decreased room and board costs (McBurney et al. 1994). This reduction in the cost of hospital care is consistant with results of subsequent studies on glutamine-enriched nutrition in post operative adults and critically ill adult and pediatric patients (Ziegler et al. 2000b).

Schloerb \& Amare (1993) studied twenty-nine patients

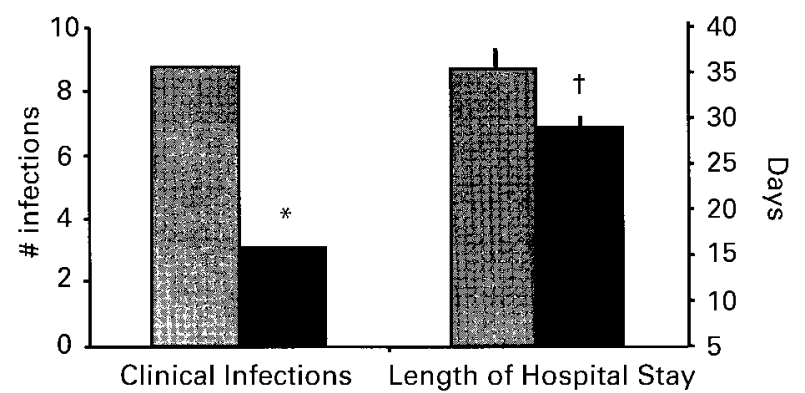

Fig. 2. L-Glutamine-supplemented parenteral nutrition decreased the incidence of clinical infection and length of hospital stay after allogeneic bone marrow transplantation. Adult patients undergoing allogeneic bone marrow transplantation (BMT) for hematologic malignancies received standard glutamine-free parenteral nutrition

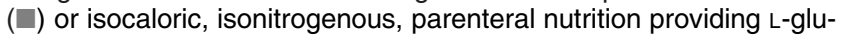
tamine $(\square)(0.57 \mathrm{~g} / \mathrm{kg}$ per day) for an average of $28 \pm 1$ and $26 \pm 2$ days after BMT. The incidence of documented clinical infections and hospital length of stay was determined. ${ }^{*} P<0.05$ and $\dagger P<0.02$ control $v$. glutamine-supplemented. after allogeneic or autologous BMT for hematologic cancer or solid tumors. In this double-blind, randomized study, similar formulations of glutamine-free and L-glutaminesupplemented PN as in the previous trial of Ziegler et al. (1992), were given for approximately 30 days. No clinically significant adverse effects related to glutamine administration were noted. The type of PN did not affect the incidence of neutrophil engraftment, mucositis, fever, clinical infection, antibiotic requirements or mortality in this BMT patient group (Schloerb \& Amare,1993). Total body water increased over time in the controls; in contrast, glutamine-treated patients exhibited a decrease in this stress-related parameter (control $+3 \cdot 1 \pm 1.5 \mathrm{v}$. glutamine $-3 \cdot 4 \pm 1 \cdot 3 \mathrm{~L} ; P<0 \cdot 05)$. As demonstrated in the earlier trial, administration of glutamine-supplemented PN significantly decreased the length of hospital stay (33 \pm 2 days v. $27 \pm 1$ days; $P<0.05$ ) (Schloerb \& Amare,1993). The patient populations investigated, clinical conditions, and cancer treatment protocols were different in these two initial studies and this may have influenced the different effect of glutamine on infection rates. A third double-blind, randomized trial suggesting that intravenous dipeptide glycyl-glutamine reduces fever and gut toxicity in unselected BMT patients has been published by another group, but only in abstract form (Poynton et al. 1995).

\section{Studies on oral administration of glutamine in BMT}

Schloerb \& Skikne (1999) recently performed a prospective, randomized double-blind trial in sixty-six cancer patients with hematological malignancies (receiving either autologous or allogeneic BMT) or solid tumors (receiving autologous BMT). Patients were randomized to either oral glutamine $(n=35)$ or glycine $(n=31)$ at a dose of $10 \mathrm{~g}$ three times daily. If PN was necessary, patients randomized to the oral glutamine group received PN supplemented with L-glutamine $(0.57 \mathrm{~g} / \mathrm{kg}$ per day) while control subjects received isonitrogenous, isocaloric glutamine-free PN. Respective comparisons were performed in patients undergoing allogeneic or autologous BMT for hematologic cancer and subjects with solid tumors undergoing autologous BMT (Schloerb \& Skikne, 1999). No significant differences were noted between study groups for hospital length of stay, days on $\mathrm{PN}$, neutrophil recovery, incidence of infection, or the incidence and severity of mucositis or diarrhea (Schloerb \& Skikne, 1999). However, in patients with hematologic malignancy $(n=43)$, glutamine administration was associated with a possible reduction in need for $\mathrm{PN}$ and a clear suggestion of improved long-term survival $(P=0.0572)$ (Schloerb \& Skikne, 1999).

In another recent prospective, randomized double-blind trial, Coghlin-Dickson et al. (2000) studied fifty-eight matched adult patients with leukemia or lymphoma receiving autologous or allogeneic BMT. Subjects received oral doses (30 g/day) of either L-glutamine or sucrose daily, beginning at the initiation of chemotherapy/TBI and continuing until hospital discharge or day 28 after the BMT. Amino acid intake was similar between groups (control $0.25 \mathrm{~g} / \mathrm{kg}$ per day $v$. glutamine $0 \cdot 29 \mathrm{~g} / \mathrm{kg}$ per day) and was well tolerated. No significant difference in neutrophil engraftment, oral mucositis, diarrhea, PN use, 
length of stay, disease relapse, or survival occurred between groups (Coghlin-Dickson et al. 2000).

\section{Effect of glutamine supplementation on mucositis after high-dose chemotherapy alone or in BMT regimens}

Mucositis or inflammation of the oral mucosa is a painful and dose-limiting complication that occurs after high-dose chemotherapy (Blijlevens et al. 2000). The management of oral mucositis and the inflammation that also affects esophageal, gastric and intestinal mucosa varies from center to center and optimal treatments are still not clarified (Biron et al. 2000; Rosenthal et al. 2000). Therapies in practice and those under investigation are directed at inflammatory, epithelial, ulcerative and healing phases of mucositis (Blijlevens et al. 2000; Biron et al. 2000; Rosenthal et al. 2000). Therapies under investigation include cryotherapy, cytoprotection (e.g. with prostaglandin $\mathrm{E}_{2}$ ), epithelial cell protective factors and agents that may accelerate epithelial restitution (growth factors) and use of antiseptics and antibiotics to decrease secondary infection of denuded mucosal (Blijlevens et al. 2000; Biron et al. 2000; Rosenthal et al. 2000).

In the four randomized controlled trials outlined above and in an additional controlled trial (Ziegler et al. 1992; Schloerb \& Amare, 1993; Schloerb \& Skikne, 1999; Coghlin-Dickson et al. 2000; Jebb et al. 1995), intravenous and/or oral L-glutamine did not influence incidence or severity of mucositis after BMT. However, other studies indicate potential benefits of oral glutamine in BMTassociated mucositis (Anderson et al. 1998a; Cockerham et al. 2000). In a randomized, prospective, double-blind clinical trial, Anderson et al. (1998a) evaluated effects of oral L-glutamine in 193 patients undergoing allogeneic or autologous BMT. Most subjects had hematologous malignancies but $32 \%$ had solid tumors and $9 \%$ were transplanted for inherited diseases. The pediatric and adult subjects were matched for clinical demographics and BMT regimen and randomized to either oral L-glutamine or oral glycine $\left(1 \cdot 0 \mathrm{~g} / \mathrm{m}^{2}\right.$ per dose $)$ to swish-and-swallow four times daily. The study amino acids were given from hospital admission until 28 days post-BMT. In patients undergoing autologous BMT $(n=87)$, glutamine use was associated with significantly less mouth pain and less difficulty eating and decreased opiate requirements (Anderson et al. 1998a). The number of patients not requiring morphine for pain was greater in the glutamine group than in controls (53 v. $31 \%$, $P=0.04)$. When morphine was given, the duration of administration was approximately $50 \%$ less with glutamine treatment ( $5 v$. 10 days, $P=0 \cdot 005)$. These beneficial effects were not observed in allogeneic BMT patients (comprised of both matched donors and unrelated donors). There were no differences between groups in graft-versus-host disease or the incidence of infections overall. However, seven viral infections developed in placebo patients versus none in those receiving glutamine (Anderson et al. 1998a). Further, in autologous BMT patients, glutamine use was associated with a significant improvement in the 28-day survival rate (100 v. $93 \%$ survival, $P=0.006$ ) but not in the 100 -day survival rate $(87 v .81 \% P=0 \cdot 18)$ (Anderson et al. 1998a).

In a recent retrospective analysis of twenty-one consecutive patients receiving high-dose paclitaxel and melphalan with autologous BMT for metastatic breast cancer, an oral L-glutamine suspension $(24 \mathrm{~g} / \mathrm{d})$ was given as a swish-and-swallow ( $4 \mathrm{~g} /$ dose 6 times daily) beginning at the onset of chemotherapy 7 days prior to BMT (Cockerham et al. 2000). Results showed that the group given glutamine demonstrated significantly fewer days of mucositis, a lower maximum grade of mucositis and required fewer days of parenteral morphine for oral pain relief (Cockerham et al. 2000).

Several studies have been published on effects of oral glutamine in non-BMT patients receiving chemotherapy alone, with mixed results. In a crossover study of patients receiving 5-fluorouracil (5-FU) for intestinal cancer, oral glutamine ( $16 \mathrm{~g} /$ day) had no measurable effect on the incidence or severity of mucositis (Jebb et al. 1994). Bozzetti et al. (1997) studied sixty-five patients with advanced breast cancer being treated with doxifluridine. Subjects received either L-glutamine orally (30 g/day) or maltodextrine as the control for eight consecutive days during intervals before chemotherapy courses. There were no differences between groups in either doxifluridineinduced diarrhea or in tumor response (Bozzetti et al. 1997). In a double-blind randomized study of patients receiving 5-FU-based chemotherapy regimens for various cancers by Okuno et al. (1999) patients received oral glutamine (4 g swish and swallow twice daily) or placebo for 14 days beginning on the first day of chemotherapy (sixty-eight patients/group). There were no significant differences or substantial trends in the mucositis scores between the two study arms (Okuno et al. 1999).

In contrast to these data, additional data indicates that glutamine administration can decrease gastrointestinal toxicity to chemotherapy in cancer patients (Anderson et al. 1998b; Muscaritoli et al. 1997; Daniele et al. 2001). In a double-blind, crossover study of twenty-four pediatric and adult patients receiving doxorubicine chemotherapy for solid cancers, Anderson et al. (1998b) showed that twicedaily oral administration of L-glutamine $\left(2.0 \mathrm{~g} / \mathrm{m}^{2}\right.$ per dose $)$ versus glycine significantly decreased the duration of mucositis and also the severity of oral pain. In an unblinded study of fourteen patients with acute leukemia receiving high-dose combination chemotherapy, oral glutamine given at a dose of $18 \mathrm{~g} /$ day begun 3 days prior to chemotherapy induction significantly decreased the duration and severity of diarrhea and requirements for antifungal agents (Muscaritoli et al. 1997). Finally, Daniele et al. (2001) recently investigated seventy chemotherapy-naive patients with colorectal cancer randomly assigned to either oral glutamine (18 g/day) or placebo before the first cycle of a five-day course 5-FU and folinic acid. Treatment was continued for 15 consecutive days starting 5 days prior to chemotherapy. Results showed that patients receiving oral glutamine had a significantly improved D-xylose absorption and cellobiose-mannitol urinary excretion, indicating improved gut absorptive and barrier function compared to controls (Daniele et al. 2001). Glutamine also significantly decreased the severity of diarrhea and the need for loperamide therapy for diarrhea. Thus, oral glutamine in this clincial setting had a protective effect on 5-FU-induced diarrhea and abnormal intestinal function following 
chemotherapy (Daniele et al. 2001). Table 2 summarizes studies on glutamine treatment in chemotherapy-induced mucositis and diarrhea.

\section{Glutamine and BMT-associated veno-occlusive disease}

Following high dose chemotherapy for BMT, patients may develop a form of hepatic failure known as veno-occlusive disease that is due to subendothelial swelling and narrowing of the central hepatic veins, with subsequent liver outflow obstruction (Wilmore et al. 1999; Brown et al. 1998). Venoocclusive disease may be fatal and appears to be related to oxygen free radical-mediated liver injury and depletion of antioxidants, including glutathione (Brown et al. 1998). In a double-blind prospective study, clinically matched BMT patients received either $50 \mathrm{~g} /$ day of dipeptide glycylglutamine intravenously or infusion of mixed nonessential amino acids from the onset of chemotherapy until discharge from the transplant unit (Brown et al. 1998). Patients in the glutamine dipeptide group demonstrated increased plasma protein $\mathrm{C}$ and albumin levels relative to the controls, suggesting preserved hepatic function and possible decreased risk of veno-occlusive disease (Brown et al. 1998). Two reported case studies have also been published suggesting that administration of intravenous + oral L-glutamine or intravenous alanyl-glutamine dipeptide combined with oral vitamin $\mathrm{E}$ diminishes the severity of post-BMT veno-occlusive disease (Nattakom et al. 1995; Goringe et al. 1998). Further studies are indicated to determine whether glutamine and vitamin $\mathrm{E}$ treatment or the administration of other antioxidants reduces the incidence or severity of veno-occlusive disease.

Table 2. Glutamine supplementation and mucositis in cancer patients

No effect of glutamine treatment:

- Mucositis in BMT: i.v. L-GLN, 30-40 g/day (Ziegler et al. 1992, Schloerb \& Amare, 1993)

- Mucositis/diarrhea in BMT: oral L-GLN, $16 \mathrm{~g} /$ day (Jebb et al. 1995)

- Mucositis/diarrhea in BMT: oral L-GLN, $30 \mathrm{~g} /$ day \pm i.v. L-GLN (Schloerb \& Skikne, 1999)

- Mucositis/diarrhea/oral food intake in BMT: oral L-GLN, $30 \mathrm{~g} /$ day (Coghlin-Dickson et al. 2000)

- Mucositis/diarrhea in acute leukemia chemotherapy: i.v. ALA-GLN, 26 g/day (van Zaanen et al. 1994)

- Diarrhea in breast cancer+doxifluridine: oral L-GLN, $30 \mathrm{~g} /$ day (Bozzetti et al. 1997)

- Mucositis with 5-FU-based chemotherapy: oral L-GLN $16 \mathrm{~g} /$ day (Jebb et al. 1994)

- Mucositis with 5-FU-based chemotherapy: oral L-GLN 8 g/day (Okuno et al.1999)

Beneficial effect of glutamine treatment:

- Mucositis in autologous BMT: oral L-GLN, $4 \mathrm{~g} / \mathrm{m}^{2}$ per day (Anderson et al. 1998a)

- Mucositis in autologous BMT for breast cancer: oral L-GLN, $24 \mathrm{~g} /$ day (Cockerham et al. 2000)

- Diarrhea after high-dose chemotherapy for leukemia: oral L-GLN, $18 \mathrm{~g} /$ day (Muscaritoli et al. 1997)

- Diarrhea and need for loperamide therapy after 5-FU therapy for colorrectal cancer: oral L-GLN, $18 \mathrm{~g} /$ day (Daniele et al. 2001)

$\mathrm{BMT}=$ bone marrow transplantation; $\mathrm{GLN}=$ glutamine; i.v. = intravenous; $5-\mathrm{FU}=5$-fluorouracil

\section{Effects of glutamine supplementation in non-BMT chemotherapy patients}

An earlier prospective, double-blind trial evaluated the efficacy of PN enriched in alanyl-glutamine dipeptide ( $40 \mathrm{~g} /$ day) versus matched control PN patients undergoing high-dose chemotherapy for acute leukemia (Van Zaanen et al. 1994). Administration of GLN dipeptide did not improve neutrophil engraftment, infection rates, use of antibiotics, mucositis, or diarrhea (Van Zaanen et al. 1994). Decker-Bauman et al. (1999) recently published an unblinded prospective study in twenty-four patients with metastatic colon cancer undergoing 5-day infusions of high-dose 5-FU. Intravenous glycyl-glutamine dipeptide $(0.4 \mathrm{~g} / \mathrm{kg}$ per day) was given to twelve patients and the twelve controls received no additional treatment. There were no significant differences between groups in the incidence or severity of clinical adverse effects during the study. However, endoscopic visual analysis and histomorphology showed that subjects receiving glutamine dipeptide demonstrated significantly decreased inflammation and ulceration of the gastric and duodenal mucosa after the third chemotherapy course and improved duodenal villus height/crypt depth ratio at this time (Decker-Bauman et al. 1999). In patients undergoing high dose cispatin/5-FU chemotherapy plus mediastinal radiotherapy for esophageal cancer, Yoshida et al. (1998) showed that orally administered glutamine ( $30 \mathrm{~g} /$ day) versus isonitrogenous, glutamine-free intravenous mixed amino acids improved circulating lymphocyte number and function and decreased gut permeability over a 28 -day period.

\section{Summary}

The administration of enteral or parenteral glutamine at doses of up to $40 \mathrm{~g} /$ day appears safe in the studies of patients receiving BMT and high-dose chemotherapy published to date. Further, several positive studies on clinical outcomes have been published in these patient groups as summarized above. Based on these data, additional double-blind, controlled clinical trials of glutamine-enriched nutrition in patients receiving BMT and chemotherapy appear indicated, particularly in light of the novel combinations of cytoreductive agents and hematopoietic growth factors now used in BMT clinical care and research.

The mechanisms of the beneficial glutamine actions observed in some studies of BMT and chemotherapy patients are unclear and additional studies to define these are needed. Glutamine supplementation exerts a number of effects that may be interrelated (Darmaun, 2000; Griffiths, 1999; Ziegler et al. 2000a; Wilmore \& Shabert, 1998; Ziegler et al. 2000b). For example, reduced protein breakdown rates may improve patient nitrogen retention. Improved resistance to colonization and decreased clinical infection may be related to enhanced number/function of tissue-associated or circulating immune cells, maintenance of gut mucosal barrier defenses, and/or improved tissue antioxidant status (Darmaun, 2000; Griffiths, 1999; Ziegler et al. 2000a; Wilmore \& Shabert, 1998; Ziegler et al. $2000 b$ ). Decreased mucositis observed in some studies may 
be related to cytoprotective or antioxidant effects of glutamine or use as a fuel source for epithelial cells.

It is possible that glutamine is utilized as a growth factor or fuel for malignant cells or may alter pharmacokinetic interactions between malignant tumors and chemotherapeutic drugs (Wilmore et al. 1999). Thus, carefully planned pharmacological studies may be needed when large doses of glutamine are given to patients receiving cytotoxic drugs. However, the available clinical data in cancer patients does not suggest that glutamine-supplemented nutrition enhances or induces tumor growth or worsens clinical outcomes. However, further study of long-term outcomes with glutamine supplementation in cancer patients, as with other novel forms of therapy, is indicated to complement primarily short-term safety data available to date. Glutamine supplementation should be considered in the design of future randomized, controlled clinical trials and in the metabolic support of individuals undergoing marrow transplantation for cancer.

\section{Acknowledgements}

Supported, in part, by National Institutes of Health grants R01 DK55850 and M01 RR00039

\section{References}

Anderson PM, Ramsay NRC, Rydholm N, Rogosheske J \& Nicklow R (1998a) Use of low dose oral glutamine in painful stomatitis during bone marrow transplantation. Bone Marrow Transplantation 22, 339-344.

Anderson PM, Schroeder G \& Skubitz KM (1998b) Oral glutamine reduces the duration and severity of stomatitis after cytotoxic cancer chemotherapy. Cancer 83, 1433-1439.

Biron P, Sebban C, Gourmet R, Chvetzoff G, Philip I \& Blay JY (2000) Research controversies in management of oral mucositis. Supportive Care in Cancer 8, 68-71.

Blijlevens NM, Donnelly JP \& De Pauw BE (2000) Mucosal barrier injury: biology, pathology, clinical counterparts and consequences of intensive treatment for haematological malignancy: an overview. Bone Marrow Transplantation 25, 1269-1278.

Bozzetti F, Biganzoli L, Gavazzi C, Cappuzzo F, Carnaghi C, Buzzoni R, Dibartolomeo M \& Baietta E (1997) Glutamine supplementation in cancer patients receiving chemotherapy: a double-blind randomized study. Nutrition 13, 748-751.

Brown SA, Goringe A, Fegan C, Davies SV, Giddings J \& Whittaker JA (1998) Parenteral glutamine protects hepatic function during bone marrow transplantation. Bone Marrow Transplantation 22, 281-284.

Cockerham MB, Weinberger BB \& Lerchie SB (2000) Oral glutamine for the prevention of oral mucositis associated with high-dose paclitaxel and melphalan for autologous bone marrow transplantation. Annals of Pharmacotherapy 34, 300-303.

Coghlin-Dickson TM, Wong RM, Negrin RS, Shizuru JA, Johnston LJ, Hu WW, Blume KG \& Stockerl-Goldstein KE (2000) Effect of oral glutamine supplementation during bone marrow transplantation. Journal of Parenteral and Enteral Nutrition 24, 61-66.

Daniele B, Perrone F, Gallo C, Pignata S, De Martino S, De Vivo R, Barletta E, Tambaro R, Abbiati R \& D'Agostino L (2001) Oral glutamine in the prevention of fluorouracil induced intestinal toxicity: a double blind, placebo controlled, randomised trial. Gut 48, 28-33.
Darmaun D (2000) Role of glutamine depletion in severe illness. Diabetes, Nutrition and Metabolism 13, 25-30.

Decker-Baumann C, Buhl K, Frohmuller S, Herbay AV, Dueck M \& Schlag PM (1999) Reduction of chemotherapy-induced sideeffects by parenteral glutamine supplementation in patients with metastatic colorectal cancer. European Journal of Cancer 35, 202-207.

Durken M, Agbenu J, Finckh B, Hubner C, Pichlmeier U, Zeller W, Winkler K, Zander A \& Kohlschutter A (1995) Deteriorating free radical-trapping capacity and antioxidant status in plasma during bone marrow transplantion. Bone Marrow Transplantation 15, 757-762.

Goringe AD, Brown S, O'Callaghan VO, Rees J, Jebb S, Elia M \& Poynton CH (1998) Glutamine and vitamin $\mathrm{E}$ in the treatment of hepatic veno-occlusive disease following highdose chemotherapy. Bone Marrow Transplantation 21, $829-832$.

Griffiths RD (1999) Glutamine: establishing clinical indications. Current Opinion in Clinical Nutrition and Metabolic Care 2, $177-182$.

Jebb SA, Marcus R \& Elia M (1995) A pilot study of oral glutamine supplementation in patients receiving bone marrow transplants. Clinical Nutrition 14, 162-165.

Jebb SA, Osborne RJ, Maughant TS \& Elia M (1994) 5-Fluorouracil and folinic acid induced mucositis: no effect of oral glutamine supplementation. British Journal of Cancer 70, $732-735$.

Jonas CR, Puckett AB, Jones DP, Griffith DP, Szeszycki EE, Bergman GF, Carlson JL, Newman MR, Galloway JR, Blumberg JB \& Ziegler TR (2000) Plasma antioxidant status after high-dose chemotherapy: A randomized trial of parenteral nutrition in bone marrow transplantation patients. American Journal of Clinical Nutrition 72, 181-189.

Klein S \& Koretz R (1994) Nutrition support in patients with cancer: What do the data really show? Nutrition in Clinical Practice 9, 91-100.

McBurney M, Young LS, Ziegler TR \& Wilmore DW (1994) A cost-evaluation of glutamine-supplemented parenteral nutrition in adult bone marrow transplantation. Journal of the American Dietetic Association 94, 1263-1266.

Mogul MJ (2000) Unrelated cord blood transplantation $v$. matched unrelated donor bone marrow transplantation: the risks and benefits of each choice. Bone Marrow Transplantation 26, Suppl 2, S58-60.

Muscaritoli M, Micozzi A, Conversano L, Martino P, Petti MC, Cartoni C, Cascino A \& Rossi-Fanelli F (1997) Oral glutamine in the prevention of chemotherapy-induced gastrointestinal toxicity. European Journal of Cancer 33, 319-320.

Nattakom TU, Charlton A \& Wilmore DW (1995) Use of vitamin $\mathrm{E}$ and glutamine in the successful treatment of severe venoocclusive disease following bone marrow transplantation. Nutrition in Clinical Practice 10, 16-18.

Okuno SH, Woodhouse CO, Loprinzi CL, Sloan JA, LaVasseur BI, Clemens-Schutjer D, Swan D, Axvig C, Ebbert LP, Tirona MR, Michalak JC \& Pierson N (1999) Phase III controlled evaluation of glutamine for decreasing stomatitis in patients receiving fluorouracil (5-FU)-based chemotherapy. American Journal of Clinical Oncology 22, 258-261.

Poynton CH, Maughan T \& Elia M (1995) Glycyl L-glutamine reduces gut toxicity in bone marrow transplantation. Blood $\mathbf{8 6}$, 586 (abstract).

Rosenthal C, Karthaus M \& Ganser A (2000) New strategies in the treatment and prophylaxis of chemo- and radiotherapy-induced oral mucositis. Antibiotics and Chemotherapy 50, 115-132.

Scheltinga MR, Young LS, Benfell K, Bye R, Ziegler TR, Santos A, Antin JH, Schloerb PR \& Wilmore DW (1991) Glutamine- 
enriched intravenous feedings attenuate extracellular fluid expansion after surgical stress. Annals of Surgery 214, 385-393.

Schloerb PR \& Amare M (1993) Total parenteral nutrition with glutamine in bone marrow transplantation and other clinical applications (a randomized doubleblind study). Journal of Parenteral and Enteral Nutrition 17, 407-413.

Schloerb PR \& Skikne BS (1999) Oral and parenteral glutamine in bone marrow transplantation: a double-blind, controlled study. Journal of Parenteral and Enteral Nutrition 23, 117-122.

Slavin S (2000) New strategies for bone marrow transplantation. Current Opinions in Immunology 12, 542-551.

Van Zaanen HCT, Van Der Lelie H, Timmer JG, Furst P \& Sauerwein HP (1994) Parenteral glutamine dipeptide supplementation does not ameliorate chemotherapy-induced toxicity. Cancer 74, 2879-2884.

Weisdorf S, Lysne J, Wind D, Haake RJ, Sharp HL, Goldman A, Schissel K, McGlave PB, Ramsay NK \& Kersey JH (1987) Positive effect of prophylactic total parenteral nutrition on longterm outcome of bone marrow transplantation. Transplantation 43, 833-838.

Wilmore DW, Schloerb PR \& Ziegler TR (1999) Glutamine in the support of patients following bone marrow transplantation. Current Opinion in Clinical Nutrition and Metabolic Care 2 , 323-327.

Wilmore DW \& Shabert JS (1998) Role of glutamine in immunologic responses. Nutrition 14, 618-626.

Yoshida S, Matsui M, Shirouza Y, Fujita H, Yamana H \& Shirouzu K (1998) Effects of glutamine supplements and radiochemotherapy on systemic immune and gut barrier function in patients with advanced esophageal cancer. Annals of Surgery 227, 485-491.

Young LS, Bye R, Scheltinga M, Ziegler TR, Jacobs DO \& Wilmore DW (1993) Patients receiving glutamine-supplemented intravenous feedings report an improvement in mood. Journal of Parenteral and Enteral Nutrition 17, $422-427$.

Ziegler TR, Bazargan N, Leader LM \& Martindale RG (2000a) Glutamine and the gastrointestinal tract. Current Opinion in Clinical Nutrition and Metabolic Care 3, 355-362.

Ziegler TR, Bazargan N \& Galloway JR (2000b) Glutamineenriched parenteral nutrition; saving nitrogen and saving money? Clinical Nutrition 19, 375-377.

Ziegler TR, Benfell K, Smith RJ, Young LS, Brown E, FerrariBaliviera E, Lowe DK \& Wilmore DW (1990) Safety and metabolic effects of L-glutamine administration in humans. Journal of Parenteral and Enteral Nutrition 14, 137S-146S.

Ziegler TR, Bye RL, Persinger RL, Young LS, Antin JH \& Wilmore DW (1998) Effects of glutamine supplementation in circulating lymphocytes after bone marrow transplantation: a pilot study. American Journal for the Medical Sciences 315, $4-10$.

Ziegler TR, Young LS, Benfell K, Scheltinga M, Hortos K, Bye R, Morrow FD, Jacobs DO, Smith RJ, Antin JH \& Wilmore DW (1992) Clinical and metabolic efficacy of glutaminesupplemented parenteral nutrition following bone marrow transplantation: A randomized, double-blind, controlled study. Annals of Internal Medicine 116, 821-828. 